\title{
Renoprotective effects of a novel inhibitor of advanced glycation
}

\author{
J. M. Forbes ${ }^{1}$, T. Soulis ${ }^{1}$, V.Thallas ${ }^{1}$, S. Panagiotopoulos ${ }^{1}$, D. M. Long ${ }^{1}$, S. Vasan ${ }^{2}$, D. Wagle ${ }^{2}$, G.Jerums ${ }^{1}$, \\ M.E.Cooper ${ }^{1}$ \\ ${ }^{1}$ Department of Medicine and Endocrine Unit, University of Melbourne, Austin and Repatriation Medical Centre \\ (Repatriation Campus), Banksia Street, West Heidelberg, Australia \\ ${ }^{2}$ Alteon Inc, 170 Williams Drive, Ramsey, New Jersey, USA
}

\section{Abstract}

Aims/hypothesis. ALT-946, an inhibitor of advanced glycation with a minimal inhibitory effect on nitric oxide synthase, was compared with aminoguanidine in experimental diabetic nephropathy.

Methods. In vitro and in vivo assays were used to assess the ability of ALT-946 to inhibit AGE-protein cross-link formation. Diabetic animals were randomly allocated into groups receiving aminoguanidine for 32 weeks, ALT-946 or vehicle (untreated). As a delayed intervention protocol, an additional diabetic group was treated with ALT-946 from week 16 to week 32 of the study. Non-diabetic rats were studied concurrently. Systolic blood pressure, body weight, plasma glucose, glycated haemoglobin and urinary albumin excretion were measured serially. Accumulation of advanced-glycation end products in the kidney was assessed by immunohistochemistry.
Results. The ALT-946 inhibitor was more potent than aminoguanidine in inhibiting AGE-protein crosslinking both in vitro and in vivo. Increased albuminuria observed in diabetic rats was attenuated in all three treatment groups. We found no difference in body weight, blood pressure or glycaemic control with any of the treatments. The untreated diabetic group had a twofold increase in glomerular staining for advanced-glycation end products compared with the diabetic groups which received treatment.

Conclusion/interpretation. ALT-946 is a potent inhibitor of advanced renal glycation end-product accumulation and reproduces the renoprotective effects of aminoguanidine. Therefore, ALT-946 should be considered as a treatment for preventing or retarding diabetic nephropathy. [Diabetologia (2001) 44: 108114]

Keywords Glycation, aminoguanidine, albuminuria, nephropathy.
It has been hypothesized that the biochemical process of advanced glycation plays an important part in the pathogenesis of diabetic vascular complications [1] and particularly in diabetic nephropathy [2]. This hypothesis was based primarily on the renoprotective effects observed with aminoguanidine (AG), an in-

Received: 17 July 2000 and in revised form: 15 September 2000

Corresponding author: Dr. J. Forbes, Department of Medicine, Repatriation Hospital, W Heidelberg 3081 Australia

Abbreviations: AER, albuminuria; AG, aminoguanidine; ALT-946, N-(2-Acetamidoethyl)hydrozinecarboximidamide hydrochloride; CML, carboxymethyllysine; INT, intervention; OD, optical density; RNase, Ribonuclease. hibitor of advanced glycation end-product formation (AGE) [3]. Aminoguanidine, however, has other actions including inhibiting nitric oxide synthase, and thus, nitric oxide production [4]. Nitric oxide has direct effects on renal haemodynamics in diabetes $[5$, 6] and therefore it has been difficult to ascertain if the benefits of aminoguanidine relate primarily to inhibiting renal AGE accumulation.

We compared the effect of AG in diabetic rats with ALT-946, N-(2-Acetamidoethyl) hydrozinecarboximidamide hydrochloride, a more potent inhibitor of AGE-derived protein modification than AG. ALT-946 is also a poor inhibitor of nitric oxide synthase with fewer toxic side effects than AG (unpublished results). We have reported previously that AG 
treatment after the development of nephropathy confers renoprotection [7]. Hence, in addition to rats treated throughout the course of diabetes, we observed a group which was treated with ALT-946 after 16 weeks of experimental diabetes. This study aims to show the efficacy of using ALT-946 in the prospective treatment of experimental diabetes and to find its potential as a late therapeutic intervention in known cases of diabetic nephropathy.

\section{Materials and methods}

In vitro assay. The ability of ALT-946 to inhibit AGE-derived protein cross-linking was compared with AG using a sandwich enzyme immunoassay. A preparation of AGE-BSA by in vitro glycation of BSA with glucose, was incubated in either the presence or absence of the test compounds on rat tail tendon collagen (TTC) coated microtitre plates (Biocoat plates purchased from Collaborative Biomedical Products, Bedford, Mass., USA). Formation of the collagen-AGE-BSA complex (TTC-AGE-BSA) was measured using rabbit-anti-BSA antibody, horseradish peroxidase-linked goat-anti rabbit IgG antibody and $\mathrm{H}_{2} \mathrm{O}_{2}$ substrate containing ABTS chromogen [8]. The optical density (OD) was measured on a Dynatech ELISA-plate reader with the sample wavelength of $410 \mathrm{~nm}$ and the reference wavelength of $630 \mathrm{~nm}$. Inhibition of cross-linking was expressed as the percentage decrease in OD when AGEBSA was incubated with TTC in the presence of ALT-946 or AG compared with the control rats treated with AGE-BSA alone. The $\mathrm{IC}_{50}$ values, defined as the concentration $(\mathrm{mmol} / \mathrm{l})$ required for $50 \%$ inhibition of TTC-AGE-BSA cross-linking, were calculated using Graphpad Inplot software, 1992 (Graphpad Software, William J. Nicklas, UMDNJ).

In vivo efficacy. We evaluated the ability of ALT-946 to inhibit AGE-derived protein cross-links that are formed in vivo during diabetes by using streptozotocin diabetic rats. Male Lewis rats weighing about $200 \mathrm{~g}$ (obtained from Charles River Company, Wilmington, Mass., USA) were used for this study. Rats were given i. v. injection of streptozotocin $(65 \mathrm{mg} / \mathrm{kg}$ in citrate buffer $\mathrm{pH} 4.5$ ). One week following the induction of diabetes, we tested blood glucose values and we only used rats with blood glucose of $15 \mathrm{mmol} / \mathrm{l}$, or more. The rats were given various daily doses of ALT-946 and AG, by oral gavage. Control rats were given only vehicle.

The in vivo efficacies of ALT-946 and AG were assessed by the decrease in $\mathrm{IgG}$ cross-linking to red blood cell membrane proteins (RBC-IgG assay) compared with the vehicle control [9].

Protocol. This study was carried out over 32 weeks because this duration of diabetes is associated with accumulation of AGE in the kidney and renal disease as assessed by functional and structural parameters [3]. Male Sprague Dawley rats weighing 200 to $250 \mathrm{~g}$ were randomly allocated into control groups or diabetic groups. The rats were rendered diabetic by intravenous injection of the beta-cell toxin streptozotocin at a dose of $55 \mathrm{mg} / \mathrm{kg}$ body weight after an overnight fast. Only rats with blood glucose concentrations of $20 \mathrm{mmol} / \mathrm{l}$ or more, with one week post-induction of diabetes, were included in the study. Diabetic rats were then further randomised to receive either AG hydrogen carbonate (Fluka Chemica, Buchs, Switzerland) at $1 \mathrm{~g} / \mathrm{l}$ in drinking water [3] or the inhibitor of AGE formation, ALT-946 (Alteon, Ramsay, N.J., USA), at a dose of $1 \mathrm{~g} / \mathrm{l}$ in drinking water. An additional group received the same dose of ALT-946 beginning at 16 weeks of diabetes and continuing for the remaining 16 weeks of the study (ALT946INT). Survival rates were improved in diabetic animals during the study period by daily treatment with $4 \mathrm{U}$ of Ultratard insulin.

Throughout the study all rats were given free access to food and water (GR2 rat cubes, Clark King and Co., Melbourne, Australia). Diabetic rats received $2 \mathrm{U}$ of ultralente insulin (U1tratard HM, Novo Industries, Bagsvaerd, Denmark) every 2 days to maintain body weight and improve survival over the study period. Rats were caged in groups of three. For collection of $24 \mathrm{~h}$ urine specimens, rats were weighed and placed in metabolic cages (Iffa Credo, L'Abresele, France) at 8 weekly intervals. Urinary albumin was measured by radioimmunoassay as described previously and expressed as $\mathrm{mg} / 24$ hours (AER) [7]. The interassay coefficient of variation was $6.5 \%$ $(n=48)$, at a concentration of $180 \mathrm{ng} / \mathrm{ml}$ and the detection limit of the assay was $31.2 \mathrm{ng} / \mathrm{ml}$. Blood was collected concurrently from the tail vein for measurement of plasma glucose by the glucose oxidase technique [10] and for measurement of glycated haemoglobin by high performance liquid chromatography (Biorad, Richmond, Calif., USA) [11]. Blood pressure was also measured every 8 weeks by tail cuff plethysmography [12]. Glomerular filtration rate (GFR) was measured by a single injection of ${ }^{99} \mathrm{Tc}-\mathrm{DTPA}$ at week 32 [11].

At week 32, rats were anaesthetized with pentobarbital sodium (Nembutal, Bomac, Asquith, Australia) and the kidneys were removed for subsequent immunohistochemistry. Formalin-fixed, paraffin embedded sections $(4 \mu \mathrm{m})$ of kidney were rehydrated and treated with $1 \% \mathrm{H}_{2} \mathrm{O}_{2}$ /methanol followed by incubation in Protein Blocking Agent (Lipshaw-Immunon, Pittsburgh, Pa., USA) for $20 \mathrm{~min}$ at room temperature. Sections were then sequentially incubated with an anti-AGE antibody (described below) for $30 \mathrm{~min}$ at room temperature, washed in phosphate buffered saline (PBS) and incubated with biotinylated goat anti-rabbit immunoglobulin (Dako, Carpinteria, Calif., USA). This was followed by another PBS wash and incubation with peroxidase conjugated streptavidin (Dako, Carpinteria). Peroxidase localisation was shown using diaminobenzidine tetrahydrochloride (DAB) as the chromogen. The AGE antibody used in this protocol has been described in detail [13]. In brief, this antibody detects advanced glycated proteins including advanced glycated BSA and RNase. Studies on the characterisation of the epitope of this antibody indicate that it detects carboxymethyllysine (CML) containing proteins but does not detect native BSA, native RNase or the AGE, pentosidine [14].

All animal procedures were carried out in accordance with the ethics protocols determined by the National Health and Medical Research Council of Australia.

Statistics. Logarithmic transformation of urinary albumin data was performed to yield a normal distribution for this parameter. The specific contributions of diabetes, time and the various drug regimens and possible interactions were assessed by analysis of variance using the Statview II Program (Brainpower, Calabasas, Calif., USA) on a Macintosh G3 personal computer (Apple, Cupertino Calif., USA). Comparisons among groups at specific time points were done by Fisher's least significant difference method. A $p$ value of less than 0.05 was considered statistically significant. All data are shown as means \pm standard error of the mean (SEM) unless otherwise specified. 


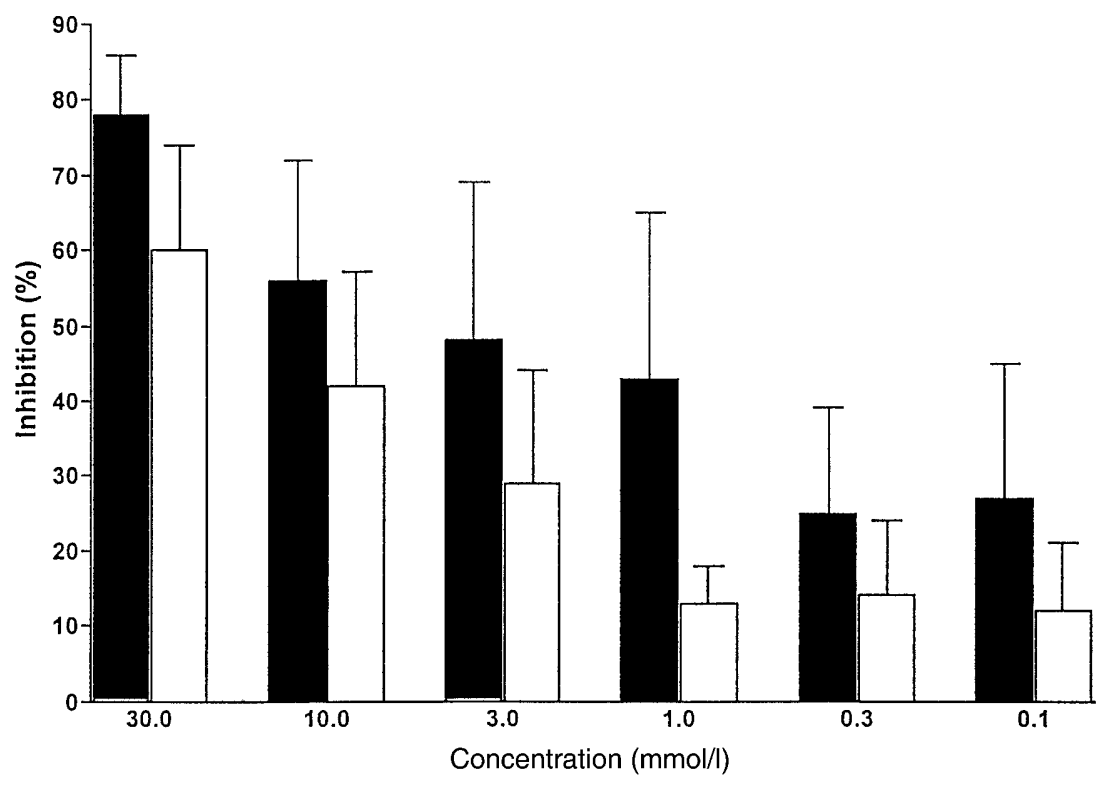

Fig. 1. Effect of ALT-946 and AG on AGE-BSA cross-linking to TTC. AGE-BSA was cross-linked to TTC in the presence and absence of ALT-946 and AG. Cross-link inhibition was calculated using the formula: $100 *$ (Mean OD control wellsMean OD test wells/Mean OD control wells). IC $_{50}$ values were calculated as described in the methods section. Filled bars ( $\square$ ) show the effect of ALT-946 and open bars ( $\square)$ show the effect of $\mathrm{AG}$

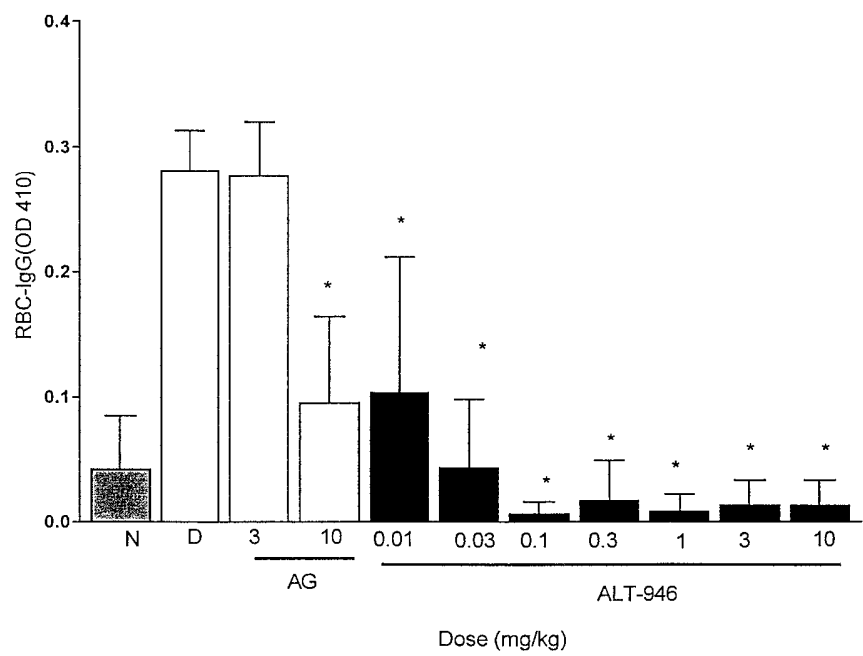

Fig. 2. Effect of ALT-946 and AG as Inhibitors of IgG Crosslinking to RBC in Diabetic Rats. Blood samples were drawn from STZ-diabetic Charles River Lewis rats after oral treatment with ALT-946, AG or vehicle alone for 8 weeks. RBCIgG levels were determined by ELISA. Decrease in RBCIgG in rats treated with the test compounds compared with the vehicle control was calculated. ${ }^{*} p<0.05$ vs diabetic rats

\section{Results}

In vitro assay. Comparisons of the inhibition of AGEBSA cross-linking to TTC at various concentrations of ALT-946 and AG are shown in Figure 1. In this assay the $\mathrm{IC}_{50}$ values of ALT-946 and AG were $3.4 \pm$ $0.1 \mathrm{mmol} / \mathrm{l}$ and $13.4 \pm 0.2 \mathrm{mmol} / \mathrm{l}$ respectively. These results indicate that in vitro ALT-946 was at least as potent if not a more potent inhibitor of AGE-protein cross-linking as AG.

In vivo efficacy. In vivo efficacy studies indicated that ALT-946 was a more effective inhibitor of AGE-protein cross-linking than AG. Figure 2 shows the RBC$\mathrm{IgG}$ levels in normal and diabetic rats and in diabetic rats after 8 weeks of daily dosing with ALT-946 or AG. The amount of IgG cross-linked to RBC was five times higher in diabetic rats compared with normal rats. Dosing with $0.03 \mathrm{mg} / \mathrm{kg}$ ALT-946 completely normalized the RBC-IgG levels in diabetic rats, whereas achieving a similar effect with $A G$ required doses in excess of $10 \mathrm{mg} / \mathrm{kg}$. These results indicate that in vivo ALT-946 was considerably more potent than $\mathrm{AG}$ for inhibition of AGE-derived protein cross-linking (Fig. 2, $p<0.05$ ).

Kidney AGEs and AER. Diabetic rats had significantly elevated plasma glucose concentrations and $\mathrm{HbA}_{1 \mathrm{c}}$ values compared with control rats (Table 1). Diabetes was also associated with reduced weight gain throughout the study period. Treatment of diabetic rats with either AG or ALT-946 did not significantly affect glycaemic control or body weight. Diabetic rats had modest although significant $(p<0.01)$ increases in mean blood pressure when compared with control rats at weeks 8 to 32 (Table 1). Systolic blood pressure among diabetic animals was not affected by any treatment. 
Table 1.

\begin{tabular}{|c|c|c|c|c|c|c|}
\hline Group & $n$ & $\begin{array}{l}\text { Body weight } \\
\text { (g) }\end{array}$ & $\begin{array}{l}\text { SB P } \\
(\mathrm{mmHg})\end{array}$ & $\begin{array}{l}\text { Plasma glucose } \\
(\mathrm{nmol} / \mathrm{l})\end{array}$ & $\begin{array}{l}\mathrm{HbA}_{1 \mathrm{c}} \\
(\%)\end{array}$ & $\begin{array}{l}\text { GFR } \\
(\mathrm{ml} / \mathrm{min})\end{array}$ \\
\hline Control rats & 10 & $729 \pm 27$ & $110 \pm 5$ & $6.3 \pm 0.1$ & $4.2 \pm 0.2$ & $3.6 \pm 0.2$ \\
\hline Diabetic rats & 16 & $346 \pm 17^{\mathrm{b}}$ & $138 \pm 7^{\mathrm{b}}$ & $20.0 \pm 1.4^{\mathrm{b}}$ & $11.5 \pm 0.3^{\mathrm{b}}$ & $4.4 \pm 0.3^{\mathrm{a}}$ \\
\hline DALT-946 0-32 & 8 & $331 \pm 22^{b}$ & $138 \pm 6^{b}$ & $22.9 \pm 0.9^{\mathrm{b}}$ & $11.4 \pm 0.3^{\mathrm{b}}$ & $3.4 \pm 0.3^{c}$ \\
\hline DALT-946 16-32 (INT) & 7 & $315 \pm 11^{b}$ & $133 \pm 7^{b}$ & $20.9 \pm 1.5^{\mathrm{b}}$ & $11.1 \pm 0.3^{\mathrm{b}}$ & $3.6 \pm 0.1^{\mathrm{c}}$ \\
\hline
\end{tabular}

${ }^{\mathrm{a}} p<0.05,{ }^{\mathrm{b}} p<0.01$ vs control rats; ${ }^{\mathrm{c}} p<0.05$ vs diabetic rats

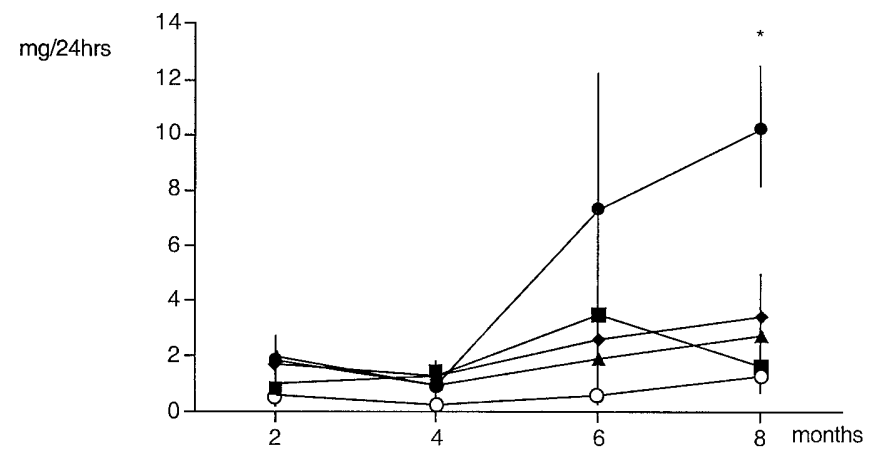

Fig.3. Serial data for albuminuria (y-axis, logarithmic scale) are shown at 8 weekly intervals for control $(\bigcirc)$, diabetic $(\mathbf{O})$, Diabetic + aminoguanidine $(\bullet)$, diabetic + ALT-946 $(\boldsymbol{\Delta})$ and diabetic + ALT-946INT ( $\boldsymbol{\square})$. Results are shown as geometric means and tolerance factors. $* p<0.05$ versus all diabetic groups, $* * p<0.05$ versus diabetic $+\mathrm{AG}$

At week 32, the GFR of diabetic rats was significantly increased compared with control rats $(p<0.05$, Table 1$)$. Treatment with either AG or ALT-946 given for 32 weeks or as a delayed intervention from weeks 16 to 32 , attenuated the increase in GFR in diabetic rats. Throughout the study, urinary albumin excretion in diabetic animals was increased when compared with control rats (Fig. 3). Before the 16-week period there was no significant effect on albuminuria by AG or any other therapy. By 32 weeks, ALT-946 had prevented the development of diabetes-associated albuminuria. In addition, delayed intervention with ALT-946 retarded the development of albuminuria at week 32. Aminoguanidine therapy also retarded the increase in urinary albumin excretion observed at 32 weeks.

Immunohistochemistry for AGE in rat glomeruli from the four study groups is shown in Figures 4 and 5. There was widespread staining for AGE in the kidney including glomeruli as well as in distal tubules and collecting ducts and to a lesser extent in proximal tubules. Diabetes was associated with increased intensity of staining, most readily detected within glomeruli (Figs. 4, 5). Treatment of diabetic rats with AG or ALT-946 prevented increases in glomerular staining for AGE observed in the diabetic animals (Figs. $4,5, p<0.01)$. The intensity of staining was reduced to a similar degree to that observed in control rats (Fig.4). Furthermore, delayed intervention with ALT-946 was also associated with reduced glomerular AGE staining (Figs. 4, 5).

\section{Discussion}

We have shown that renal AGE accumulation observed in diabetes, can be prevented by the novel inhibitor of AGE formation, ALT-946. The ALT-946 inhibitor prevented, not only glomerular AGE accumulation but also the development of albuminuria. In vitro studies have indicated that this compound is a potent inhibitor of $\mathrm{AGE}$ accumulation equal to AG, with little inhibitory effect on inducible nitric oxide synthase. Furthermore, in vivo studies showed that this compound was even more potent than AG in preventing AGE-protein cross-linking.

The importance of inhibiting AGE accumulation has recently been shown using other inhibitors of advanced glycation. In one study on spontaneous diabetes, a thiazolidine derivative (OPB 9195) with the additional inhibition of AGE formation prevented renal structural injury [15] and reduced cytokine expression [16] in the OLETF rat. We have recently observed an association between vascular AGE accumulation and mesenteric vascular hypertrophy using the AGE inhibitor, 2, 3 DAP [17]. Unfortunately, that compound has failed preclinical toxicity studies and therefore other compounds need to be investigated. Other studies have led to the development of novel classes of potent, specific inhibitors of AGE formation $[8,18]$. The ALT-946 inhibitor is derived from that pharmaceutical programme and has been shown by this in vivo study to be a potent inhibitor of renal AGE accumulation.

We have shown in a previous study that renal AGE accumulation is a dynamic process that can be reversed by delayed intervention with aminoguanidine [19]. A similar finding was observed in this study, with delayed treatment with ALT-946 leading to reduced glomerular AGE accumulation. There was no difference observed between delayed intervention with ALT946 and continuous treatment throughout the duration of diabetes. The effects on glomerular AGE accumulation were associated with 

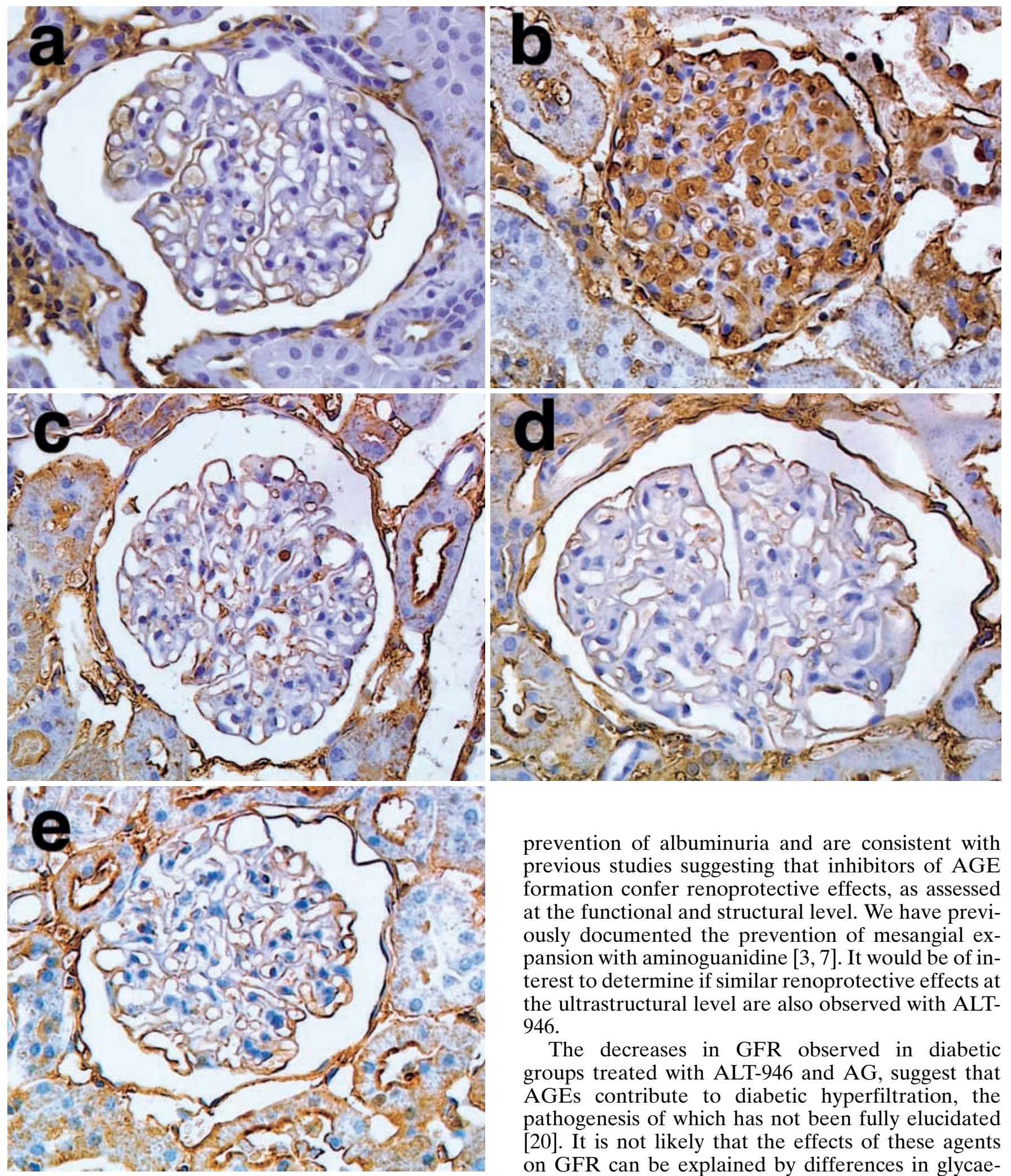

Fig. 4. Immunohistochemical staining for AGE in the kidney is shown for (A) control; (B) diabetic; $(\mathbf{C})$ diabetic $+\mathrm{AG}$; (D) diabetic + ALT-946; (E) diabetic + ALT-946INT. Magnification $\times 400$

prevention of albuminuria and are consistent with previous studies suggesting that inhibitors of AGE formation confer renoprotective effects, as assessed at the functional and structural level. We have previously documented the prevention of mesangial expansion with aminoguanidine [3,7]. It would be of interest to determine if similar renoprotective effects at the ultrastructural level are also observed with ALT946.

The decreases in GFR observed in diabetic groups treated with ALT-946 and AG, suggest that AGEs contribute to diabetic hyperfiltration, the pathogenesis of which has not been fully elucidated [20]. It is not likely that the effects of these agents on GFR can be explained by differences in glycaemic control because none of the treatments influenced plasma glucose, body weight or glycated haemoglobin. Other investigators have reported that early glycation products induce glomerular hyperfiltration [21] but it was difficult to determine how accurately early AGEs had been separated from late AGEs in this study. This indicates that direct effects 


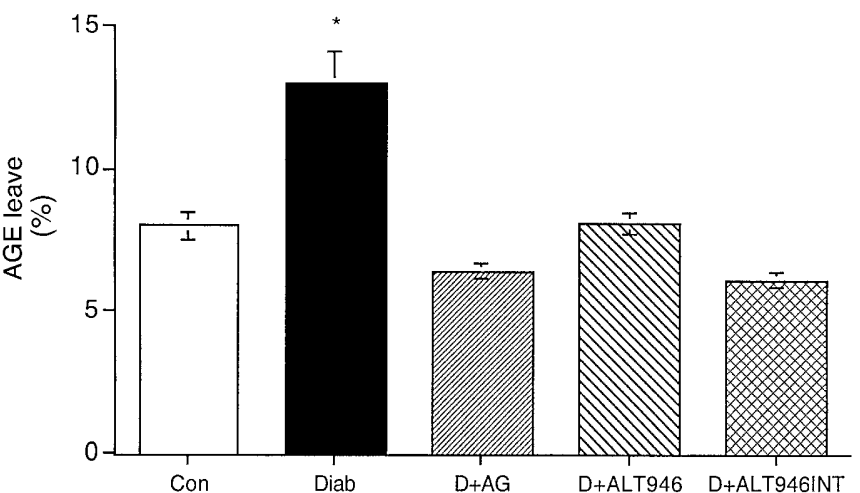

Fig.5. Quantification of glomerular advanced glycation end products (\% glomerular area) is shown as means \pm SEM. $* p<0.01$ vs all groups

of AGEs on glomerular filtration cannot be excluded.

Possibly, ALT-946 is also relevant not only to renal disease, but to other vascular complications observed in diabetes. Aminoguanidine has been shown to have retinoprotective [22] and vasoprotective effects [23]. A role for ALT-946 in vasculopathy, however, remains to be shown. Evidence provided by other inhibitors of AGE such as 2, 3 DAP, without significant inhibitory activity agent nitric oxide synthase, have been reported to attenuate diabetes associated vascular hypertrophy [17], a phenomenon which seems to be partly AGE dependent [23]. Therefore, the effectiveness of ALT-946 in vasculopathy should be investigated further.

We have shown that prevention of AGE formation by ALT-946 without inhibition of nitric oxide synthase reproduces the protective effects of AG. Other agents which inhibit nitric oxide synthase without effects on AGE formation such as L-NAME and methylguanidine have failed to confer similar renal protection observed with AG [13]. It is therefore not likely that the nitric oxide inhibitory action of the $\mathrm{AG}$ is mediating the renoprotective effect of this agent.

This study provides preliminary evidence suggesting that agents such as ALT-946 have a role in the treatment of diabetic nephropathy and possibly diabetic vascular complications. The ALT-946 inhibitor is a more potent and selective inhibitor of AGE formation, with less toxicity than AG.

Acknowledgements. The authors wish to thank K. Goldring for her technical assistance. This work was supported by grants from Juvenile Diabetes Foundation International, the National Health and Medical Research Council of Australia and Alteon Corporation.

\section{References}

1. Brownlee M (1995) Advanced protein glycosylation in diabetes and aging. Annu Rev Med 46: 223-234

2. Vlassara H (1996) Protein glycation in the kidney: role in diabetes and aging. Kidney Int 49: 1795-1804

3. Soulis-Liparota T, Cooper M, Papazoglou D, Clarke B, Jerums G (1991) Retardation by aminoguanidine of development of albuminuria, mesangial expansion, and tissue fluorescence in streptozocin-induced diabetic rat. Diabetes 40: $1328-1334$

4. Corbett JA, Tilton RG, Chang K, et al. (1992) Aminoguanidine, a novel inhibitor of nitric oxide formation, prevents diabetic vascular dysfunction. Diabetes 41: 552-556

5. Bank S, Aynedjian H (1993) Role of EDRF (Nitric oxide) in diabetic renal hyperfiltration. Kidney Int 43: 1306-1312

6. Komers R, Allen TJ, Cooper ME (1994) Role of endothelium-derived nitric oxide in the pathogenesis of the renal hemodynamic changes of experimental diabetes. Diabetes 43: $1190-1197$

7. Soulis T, Cooper ME, Vranes D, Bucala R, Jerums G (1996) The effects of aminoguanidine in preventing experimental diabetic nephropathy are related to duration of treatment. Kidney Int 50: 627-634

8. Vasan S, Netram K, Zhang X, Wagle D, Ramdass R, Shih D (1996) Identification of new inhibitors of AGE-protein crosslinking using a novel ELISA. Diabetes 45 [Suppl 2]: 258 (Abstract)

9. Vasan S, Zhang X, Zhang X, et al. (1996) An agent cleaving glucose-derived protein crosslinks in vitro and in vivo. $\mathrm{Na}$ ture 382: 275-278

10. Schmidt F (1961) Enzymatic determination of glucose and fructose simultaneously. Klin Woch 39: 1244-1247

11. Allen TJ, Cooper ME, O'Brien RC, Bach LA, Jackson B, Jerums G (1990) Glomerular filtration rate in streptozocin-induced diabetic rats. Role of exchangeable sodium, vasoactive hormones, and insulin therapy. Diabetes 39: 1182-1190

12. Bunag R (1973) Validation in awake rats of a tail-cuff method for measuring systolic pressure. J Appl Physiol 34: 279-282

13. Soulis T, Cooper ME, Sastra S, et al. (1997) Relative contributions of advanced glycation and nitric oxide synthase inhibition to aminoguanidine-mediated renoprotection in diabetic rats. Diabetologia 40: 1141-1151

14. Sell DR, Lapolla A, Odetti P, Fogarty J, Monnier VM (1992) Pentosidine formation in skin correlates with severity of complications in individuals with long-standing IDDM. Diabetes 41: 1286-1292

15. Nakamura S, Makita Z, Ishikawa S, et al. (1997) Progression of nephropathy in spontaneous diabetic rats is prevented by OPB-9195, a novel inhibitor of advanced glycation. Diabetes 46: 895-899

16. Tsuchida K, Makita Z, Yamagishi S, et al. (1999) Suppression of transforming growth factor beta and vascular endothelial growth factor in diabetic nephropathy in rats by a novel advanced glycation end product inhibitor, OPB9195. Diabetologia 42: 579-88

17. Soulis T, Sastra S, Thallas V, et al. (1999) A novel inhibitor of advanced glycation end-product formation inhibits mesenteric vascular hypertrophy in experimental diabetes. Diabetologia 42: 472-479

18. Kochakian M, Manjula BN, Egan JJ (1996) Chronic dosing with aminoguanidine and novel advanced glycosylation end product formation inhibitors ameliorates cross-linking of tail tendon collagen in streptozotocin-induced diabetic rats. Diabetes 45: 1694-1700 
19. Soulis T, Cooper ME, Sastra S, Panagiotpoulos S, Jerums G (1995) Are the renoprotective effects of aminoguanidine in diabetes mediated via its action as a NO synthase inhibitor? Diabetologia 38 [Suppl 1]: A46

20. Anderson S, Vora JP (1995) Current Concepts of Renal Hemodynamics in Diabetes. Journal of Diabetes \& its Complications 9: 304-307

21. Sabbatini M, Sansone G, Uccello F, Giliberti A, Conte G, Andreucci VE (1992) Early glycosylation products induce glomerular hyperfiltration in normal rats. Kidney Int 42: 875-881
22. Hammes H, Martin S, Federlin K, Geisen K, Brownlee M (1991) Aminoguanidine treatment inhibits the development of experimental diabetic retinopathy. Proc Natl Acad Sci USA 88: 11555-11558

23. Rumble JR, Cooper ME, Soulis T, et al. (1997) Vascular hypertrophy in experimental diabetes: role of advanced glycation end products. J Clin Invest 99: 1016-1027

24. Soulis LT, Cooper ME, Dunlop M, Jerums G (1995) The relative roles of advanced glycation, oxidation and aldose reductase inhibition in the development of experimental diabetic nephropathy in the Sprague-Dawley rat. Diabetologia 38: $387-394$ 